\section{E-201 OUTCOMES OF FLOW DIVERSION OF MIDDLE AND ANTERIOR CEREBRAL ANEURYSMS AND THE INCIDENCE OF NEO-INTIMAL HYPERPLASIA}

G Kaur*, K Dakay, C Gandhi, F Al-Mufti, J Santarelli. Neurosurgery, Westchester Medical Center, Valhalla, NY

\subsection{6/neurintsurg-2020-SNIS.232}

Introduction Flow diversion of distal aneurysms like the middle cerebral artery (MCA) and anterior cerebral artery (ACA) is a safe and effective treatment modality for wide necked aneurysms, not amenable to any other endovascular approach. We present our experience with flow diversion of distal vasculature and the outcomes.

Materials and Methods Distal aneurysms were defined as MCA and ACA aneurysms. All distal aneurysm treatments performed with a Pipeline embolization device (PED) from September 2017 to January 2020 were collected in a prospectively maintained database. Patient demographics, clinical, and angiographic outcomes including digital subtraction angiography were registered. 6 month follow up angiograms were performed when possible to look for resolution of the aneurysm and the incidence of neo-intimal hyperplasia.

Results A total of 36 distal aneurysms were treated by pipeline flow diversion, of which 19 were MCA aneurysms and the rest were ACA aneurysms. Mean age is $58 \pm 11$ years. 6 month follow up is available for 21 of the 36 treated cases, with 3 being lost to follow up and the rest awaiting 6 month follow ups. From the follow ups available $(n=21)$, the incidence of complete aneurysmal resolution was $76 \%$ (16/21). Neo-intimal hyperplasia was seen in 52\% (11/21), and all cases with intimal hyperplasia were asymptomatic and had complete aneurysmal resolution. All cases of neo-intimal hyperplasia were mild to moderate and were managed by continuing dual antiplatelet therapy for another 6 months. There were 2 complications in the entire cohort- 1 intracerebral hemorrhage and 1 groin pseudo-aneurysm. There was 1 asymptomatic pipeline occlusion with no clinical manifestations.

Conclusion In our experience, flow diversion of distal wide necked MCA and ACA aneurysms is a safe and effective treatment strategy. The presence of neo-intimal hyperplasia after flow diversion of distal aneurysms is a frequent finding at 6 month follow up angiography but has been clinically asymptomatic in all cases.

Disclosures G. Kaur: None. K. Dakay: None. C. Gandhi: None. F. Al-Mufti: None. J. Santarelli: None.

\section{E-202 CREATION OF A LARGE ANIMAL ANEURYSM MODEL FOR NEUROCURE LIQUID EMBOLIC ASSESSMENT}

${ }^{1} \mathrm{~T}$ Becker*, ${ }^{1} \mathrm{~W}$ Merritt, ${ }^{1} \mathrm{C}$ Settanni, ${ }^{2} \mathrm{~N}$ Norris, ${ }^{3} \mathrm{~A}$ Ducruet, ${ }^{3} \mathrm{M}$ Preul. ${ }^{1}$ Northern Arizona University, Flagstaff, $A Z ;{ }^{2}$ Aneuvas Technologies, Inc., Flagstaff, $A Z ;{ }^{3}$ Barrow Neurological Institute, Phoenix, AZ

\subsection{6/neurintsurg-2020-SNIS.233}

Introduction We developed a clinically relevant in vivo animal model of large, wide-neck aneurysm morphologies to study aneurysms with traditionaly-high recanalization rates (up to $70 \%$ recurrence) post-treatment. This model will be treated with a new liquid embolic device under development: NeuroCURE (Aneuvas Technologies, Inc. (ATI), Flagstaff, AZ). NeuroCURE $^{\circledR}$ is delivered using dual-microcatheter techniques (device delivery behind temporary balloon/stent protection). NeuroCURE is an elastic gel material that can completely fill and stabilize aneurysms long-term, potentially reducing the recanalization risk of larger aneurysms. High recanalization rates of current endovascular devices can be partially attributed to inadequate modeling of larger aneurysms during preliminary device testing in animal models, resulting in incomplete data sets prior to device approval.

Materials and Methods The large animal model was developed in canines by the Neurosurgery Research Center at Barrow Neurological Institute (BNI) and ATI. Both swine and canines exhibit adequate blood-flow, blood pressure, and vessel size to accommodate larger aneurysm models and dual microcatheter techniques - which are not feasible in the current rabbit-elastase models. Canines have been adopted over swine because they exhibit a healing response comparable to humans. Swine exhibit an overly-aggressive clotting cascade.A lateral wall aneurysm was surgically created by anastomosis of an external jugular vein (EJV) segment onto the common carotid artery (RCCA) in the neck. The EJV segment was sewn along circular sidewall cuts $(5-7 \mathrm{~mm}$ diameter) in the RCCA to form a wide-neck aneurysm. The distal EJV was tied off at a dome height $\geq 10 \mathrm{~mm}$. The animals were survived at least 2 weeks pre-embolization.

Results Angiographic imaging verified the model forms into a patent aneurysm with large dome and wide-neck morphologies $(<2: 1$ midline-dome:neck (D:N) ratio - figure 1A). Histology verifies healing of the aneurysm neck (re-established endothelial layer). The 2-week survival prior to device treatment allows for differentiation of the model versus the device healing response for short- and long-term studies.

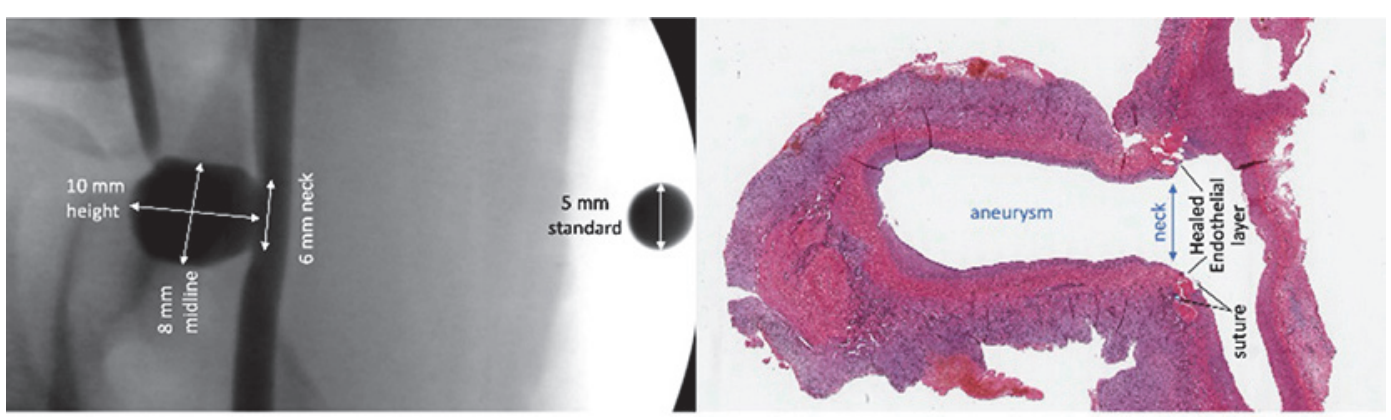

Abstract E-202 Figure 1 A Large, wide-neck, lateral-wall aneurysm model in canine, B. histology of aneurysm surgery survived 2 weeks preembolization. Endothelial layer at the neck has healed 
Conclusion By building upon previous animal modeling experiences with rabbit, swine, and canine, we are able to develop a specific model for the simulation of endovascular techniques and device deployment in large aneurysm morphologies. This model is currently being used to test the liquid embolic, Neu$\operatorname{roCURE}^{\circledR}$. The model has been approved by the Food and Drug Administration (FDA) for NeuroCURE assessment, with the goal of reducing large aneurysm recurrence rates in patients.

Disclosures T. Becker: 1; C; NIH STTR Phase II (2R42NS097069-02A1). 2; C; Aneuvas Technologies, Inc. 4; C; Aneuvas Technologies, Inc. 6; C; Aneuvas Technologies, Inc. W. Merritt: 2; C; Aneuvas Technologies, Inc. C. Settanni: 1; C; IH STTR Phase II (2R42NS097069-02A1). N. Norris: 1; C; IH STTR Phase II (2R42NS097069-02A1). A. Ducruet: 1; C; IH STTR Phase II (2R42NS097069-02A1). 4; C; Aneuvas Technologies, Inc. M. Preul: 1; C; NIH STTR Phase II (2R42NS097069-02A1). 4; C; Aneuvas Technologies, Inc.

\section{E-203 SHEATHLESS LOW-PROFILE RADIAL ACCESS UTILIZING THE 071 BENCHMARK GUIDE CATHETER FOR COMPLEX ANTERIOR \& POSTERIOR CEREBROVASCULAR INTERVENTIONS: HIGH TECHNICAL SUCCESS/LOW COMPLICATIONS WITH 'RULE OF .8'}

S Satti ${ }^{*}$, T Eden. Neurointerventional Surgery, Christiana Care Health System, Newark, DE

10.1136/neurintsurg-2020-SNIS.234

Introduction/Purpose Two factors limiting first line radial access for complex cerebrovascular interventions include: radial artery diameter and available catheter technology. Our rule for patient selection is to maintain a radial artery/sheath ratio of $\geq .8$, which we used as the primary inclusion criteria for all procedures. We have increasingly attempted sheathless radial access allowing for largest inner and smallest outer diameter. We describe a low cost/simplified approach to direct radial access with a commonly available guide catheter. We then present our experience of transradial cerebrovascular interventions comparing outcomes between procedures performed using a 6F Terumo Slender Sheath/Benchmark guide (OD 2.53 $\mathrm{mm})$ versus direct sheathless radial access with the Benchmark (OD $2.0 \mathrm{~mm}$ ).

Materials and Method Single institution retrospective electronic query of all $6 \mathrm{~F}$ radial access procedures performed between October 2010 and March 2020 access for cerebrovascular intervention using a Penumbra Benchmark 6F guiding catheter. The procedures were divided in Group 1 - 133 pts (sheath) and Group 2- 32 patients (sheathless). Summary Technique for Direct/Sheathless Access

1. Ultrasound guided: Target vessel selection/measurement. "Inclusion: artery diameter/sheath diameter $\geq .8$ )

4F micropuncture/21 g needle: Radial angiogram and Radial cocktail Exchange: Micropuncture sheath/sheathless Benchmark/BER over $.035 \times 180 \mathrm{~cm}$ glidewirePrimary outcomes included: failure of primary access (need to convert to femoral access) and major complications requiring surgery or transfusion. Secondary outcomes included: infection, development of AV fistula, or development of pseudoaneurysm.

Results Procedural success (completion of planned procedure) was achieved in $98.2 \%$ of patients (average of groups 1 and 2), no significant difference. Procedures performed included a wide spectrum of intracranial pathologies, however, aneurysm treatment represented $>60 \%$ of procedures. Success with the primary access site cannulation was $100 \%$ for both Group 1 and Group 2, when using rule of $\geq .8$. No major complications were encountered in either group of patients; no patients required transfusion or emergency vascular surgery for vessel injury/avulsion, digital ischemia, or compartment syndrome. One patient was identified in Group 1 with a symptomatic radial artery-vein fistula underwent successful elective out-patient surgical repair at 12 months.

Conclusion Sheathless radial access using a Penumbra Benchmark 071 guiding catheter can be used for cerebrovascular interventions in both anterior and posterior circulation pathology. High technical success completing index procedure, success with arterial cannulation and low complication rates may be attributed to patient selection/technique (maintaining artery/ sheath ratio of $\geq .8$ ). Direct radial access requires smaller vessel diameter potentially expanding eligible patients with smaller radial artery diameters.

Disclosures S. Satti: None. T. Eden: None.

\section{E-204 NOVEL BLOOD ANALOGUE FOR IN-VITRO NEUROVASCULAR MODELING}

H Sodawalla*, W Merritt, T Becker. Bioengineering Program, Northern Arizona University, FLAGSTAFF, AZ

\subsection{6/neurintsurg-2020-SNIS.235}

Introduction In-vitro models for simulating surgical procedures is a cost-effective way of creating fail-safe surgical protocols. Insights gained from these models can also aid in testing new medical devices and reduce the use of animal models. Traditional glass- and silicone-based in-vitro models use distilled water with surfactant additives for lubricity. However, a new material, carboxymethyl cellulose (CMC) dissolved in distilled water, can mimic the mechanical and rheological properties of blood. We have shown that CMC fluid out-performs distilled water, glycerol mixtures, and bovine blood, in terms of physiological accuracy.

Methods We tested the density and dynamic viscosity of the following blood analogs: CMC, distilled water, glycerol mixture (22 wt \%), bovine blood (Bos taurus). For each material, samples were tested with a $20 \mathrm{~mm}$ cylindrical parallel plate head geometry $(0.25 \mathrm{ml})$ attached to a hybrid rheometer (DHR-2, TA Instruments), set at $37^{\circ} \mathrm{C}$ (human physiological temperature). Three samples of each blood analog were tested and repeated 5 times. A shear rate sweep from 15 to 105 1/s was tested to cover a wide range of physiological blood flow rates. 